\title{
MODA Y SALUD EN PERSONAS MAYORES. REVISIÓN BIBLIOGRÁFICA ENFERMERA
}

\author{
Mạ Carmen Otero López \\ Centro de Salud Sayago. Máster en investigación en medicina clínica \\ carmenoterozamora@gmail.com \\ María Ballesteros Mantecón \\ Enfermera de Atención Especializada de la Unidad de UVI \\ del Hospital Virgen de la concha de Zamora \\ Ma Milagros García Álvarez \\ Enfermera Especialista en Geriatría. Enfermera de Atención \\ Especializada de la Unidad de la UCA del Complejo Asistencial de Zamora \\ Antonia Otero López \\ Diplomada en Enfermería por la Universidad de Palencia \\ Enfermera en el Centro de Salud Sanabria, Zamora \\ Sonia Pérez Martín \\ Enfermera de Atención Especializada de la Unidad de \\ Neuro - hematología del Hospital Virgen de la Concha de Zamora

\section{$\mathbf{M}^{\mathrm{a}}$ del Carmen San Raimundo Morín} \\ Enfermera de Atención Especializada de la Unidad de \\ ORL - Urología del Hospital Virgen de la concha de Zamora \\ Casandra García Otero \\ Técnico de Farmacia en el Complejo Asistencial de Zamora \\ Josefa González Centeno \\ Enfermera de atención Especializada de la Unidad de \\ Quirófano del Hospital Provincial de Zamora
}

Recepción Artículo: 16 marzo 2021 Admisión Evaluación: 16 marzo 2021 Informe Evaluador 1: 20 marzo 2021 Informe Evaluador 2: 24 marzo 2021 Aprobación Publicación: 26 marzo 2021

\section{RESUMEN}

La llamada "tercera edad" es una fase de la vida, marcada por prejuicios. La imagen corporal es la representación del cuerpo que cada persona construye en su mente, influenciada por cambios psicológicos. La ropa cambia nuestra manera de pensar y podemos sentirnos superhéroes solamente cambiando la vestimenta. Hablar de moda en la tercera edad, rompiendo los cánones de belleza preestablecidos, es extraño, la "arruga no es bella", sin embargo, como consumidores, son un 


\section{MODA Y SALUD EN PERSONAS MAYORES. REVISIÓN BIBLIOGRÁFICA ENFERMERA}

grupo activo, y se convertirán en el motor de la llamada silver economy, ya que "Son personas activas a las que les gusta cuidarse, hacer deporte, comer bien, ir a la moda y divertirse". Sentirse guapa influye en la percepción de la salud, es decir sentirse bien por fuera hace que se sienta bien por dentro. El objetivo de nuestro estudio es evaluar la influencia de la moda en las personas mayores como potenciador de la autoestima, por lo tanto, como mejora de su salud y hacer de Zamora un referente de silverización del sector de la moda. (Espacio fashion week de la moda Silver). Metodología: revisión de la literatura.

Resultados alcanzados: las personas mayores son un mercado en aumento, con rentas considerables, buena salud, interés por disfrutar, ilusiones y una estructura de consumo característica, se debería considerar sus exigencias a la hora de diseñar y comercializar haciendo una moda cómoda y funcional. Discusión: son pocos los resultados obtenidos; ante este hecho vemos que es un tema novedoso. Es un tema a desarrollar en nuevas investigaciones. Conclusión: el escaparate que vemos de las personas, es en principio, como va vestida; la forma de combinar los colores, de utilizar los complementos, se sentirse cómoda y elegante, influye sobre la percepción de la salud.

Palabras clave: vestuario; anciano; Anciano de 80 o más Años; autoimagen

\section{ABSTRACT}

Fashion and health in the elderly. nursing literature review. The so-called "third age" is a phase of life, marked by prejudices. Body image is the representation of the body that each person builds in their mind, influenced by psychological changes. Clothes change our way of thinking and we can feel like superheroes only by changing clothes. Talking about fashion in the elderly, breaking the pre-established beauty canons, it is strange, the "wrinkle is not beautiful", however, as consumers, they are an active group, and they will become the engine of the so-called silver economy, since "They are active people who like to take care of themselves, play sports, eat well, be fashionable and have fun." Feeling beautiful influences the perception of health, that is, feeling good on the outside makes you feel good on the inside. The objective of our study is to evaluate the influence of fashion in older people as an enhancer of self-esteem, therefore, as an improvement in their health and to make Zamora a benchmark for the silverization of the fashion sector. (Silver fashion week space).

Methodology: review of the literature.

Results achieved: the elderly are a growing market, with considerable incomes, good health, interest in enjoying themselves, illusions and a characteristic consumption structure, their demands should be considered when designing and marketing making a comfortable and functional fashion.

Discussion: few results have been obtained; Given this fact, we see that it is a novel topic. It is a topic to be developed in new research.

Conclusion: the showcase that we see of people is, in principle, how they are dressed; the way to combine colors, to use accessories, to feel comfortable and elegant, influences the perception of health.

Keywords: clothing; aged; Aged, 80 and over; self concept.

\section{ANTECEDENTES DE LA TEMÁTICA A TRATAR}

Se espera que para 2050, la población mundial de mayores de 60 años de edad llegue a los 2000 millones, produciéndose un aumento de 900 millones de personas comparándolo con 2015. Vivimos más años en todo el mundo, por lo tanto más tiempo (1).

El creciente aumento de personas mayores en la población actual es un cambio significativo en nuestra sociedad. El envejecimiento de la población es una característica común en Europa y en los 
países desarrollados en general, hecho causado por la disminución de la tasa de natalidad y por el incremento de la población mayor como consecuencia de una mayor esperanza de vida (2).

No hay un modelo de persona mayor «diseñado», podemos encontrar personas mayores que tienen unas facultades físicas y psíquicas muy buenas, que no tienen nada que envidiar al grupo de personas de veinte 0 treinta años, mientras que otras personas, sufren un deterioro considerable a edades mucho más tempranas (1).

La llamada "tercera edad" es una fase de la vida, marcada por prejuicios que limita la manera de actuar de la persona mayor, produciendo un deterioro de la autoestima de estas personas (3).

La autoestima 0 autoimagen es la valoración que tiene uno de sí mismo y es la que determina nuestro comportamiento en el entorno social y laboral. Las personas con una autoestima alta se enfrentan a los problemas y los desafíos con mayor seguridad (4). Quererse a uno mismo no tiene edad y es bueno realizarlo durante toda la vida, pero a medida que envejecemos perdemos la autoconfianza, ya sea por soledad 0 abandono (4).

La autoestima no está relacionada con la edad cronológica, pero a los mayores les resulta más difícil mantener una adecuada integración social y además experimentan una disminución en la capacidad de afrontamiento de los eventos cotidianos (5).

La imagen corporal es definida como la representación del cuerpo que cada persona construye en su mente (6), basada en un concepto subjetivo, multifacético e interrelacionado con los sentimientos de autoconciencia. Está fuertemente influenciada por la sociedad y la cultura en que la persona vive, así como por los valores de su entorno (7). La imagen corporal que la persona tiene sobre sí misma se ve influenciada por los cambios psicológicos $(8,9)$ que van asociados a la senectud como la depresión y trastornos de ansiedad (8) suponiendo una mayor dificultad para la adaptación a los cambios $(8,9)$, si a ello le unimos la dependencia o la ayuda, la autoimagen es más distorsionada y negativa $(8,9)$.

Sentirse guapa influye en la percepción de la salud, es decir sentirse bien por fuera hace que se sienta bien por dentro.

La imagen que proyecta una persona es fundamental para su dignidad y para que se integren de manera natural en su entorno. Verse en el espejo y estar a gusto con la imagen que se ve, refuerza la autoestima y levanta el ánimo (10).

La investigadora de la Universidad de Hertfordshire (Inglaterra) Karen Pine asegura que la ropa que llevamos cambia nuestra manera de pensar y de percibirnos a nosotros mismos nos podemos sentir superhéroes solamente cambiando la vestimenta (11).

Hablar de moda en la tercera edad, es raro, estableciendo otros prototipos de belleza y rompiendo con los preestablecidos de cuerpos delgados, piernas kilométricas y tez de porcelana (11).

La diseñadora de moda Elisa Morató asegura que la vestimenta debe adaptarse al estilo personal y debe ser funcional, cómoda y duradera. Asimismo, utilizar un tipo de ropa u otra no deberá depender de la edad que tengamos, sino de nuestros gustos, características físicas y la forma de ser (12).

La industria de la moda ha empezado a dar pequeñas demostraciones de su aprobación a esta tendencia social de la moda en personas mayores (12), la pirámide de la población, en España al igual que en otros países, se está invirtiendo y ya son más las personas mayores de 60 años que los niños (1). Empiezan a surgir supermodelos que más allá de la jubilación son todo un icono de belleza, como por ejemplo Daphne Selfe, de 92 años que (12), en la última década ha posado para la firma de gafas OPSM, para Vogue Japón, Harper's Bazaar, Tank Magazine, el suplemento de The Daily Telegraph e incluso ha sido imagen de la marca de maquillaje Eyeko London y ha aparecido en el catálogo de Primark. Dolce \& Gabbana la fichó para su campaña de 2012 (13) o Iris Apfel, a quién la fama le llegó a los 84 años gracias a la moda (12). 


\section{MODA Y SALUD EN PERSONAS MAYORES. REVISIÓN BIBLIOGRÁFICA ENFERMERA}

Es novedosa la inclusión de la tercera edad en el mundo de la moda. Sin embargo, la vejez empezó a ganar terreno de forma sorpresiva en el mundo de la moda y el concepto general sobre la vestimenta para gente mayor ya no es solo vestir prendas claras y sobrias (12).

Las personas mayores no son tenidas en cuenta como grupo de consumidores y con frecuencia son clasificadas como personas con mala salud y escasez de recursos económicos sufriendo la discriminación basada en antiguos estereotipos como la "arruga no es bella" (2).

El actual grupo de 60 a 70 años, tienen características sociodemográficas que los definen como un grupo de consumidores importante, con rentas considerables, buena salud, interés por disfrutar, ilusiones y una estructura de consumo característica, el mercado tiene que dirigirse a ellos y considerar sus exigencias a la hora de diseñar y comercializar un determinado producto (2).

Las personas mayores, como consumidores, son un grupo activo, involucradas en las compras y tendencias, tienen unos criterios de compra bien definidos por lo que conocen exactamente cuáles son sus requisitos y en qué aspectos de un producto se van a fijar a la hora de realizar la compra (2).

Los principales problemas que encuentran las personas mayores con la indumentaria es la falta de adecuación de los productos a sus necesidades, características y preferencias. Aumentando la usabilidad del producto y orientando su diseño a las personas mayores, se beneficia también el resto de la población (2).

Las formas de consumo se deberían de modificar ya que los mayores se convertirán en el motor de la llamada silver economy. (14).

La silver economy 0 economía plateada incluye todas aquellas actividades económicas, productos y servicios destinados a satisfacer las necesidades de los mayores de 50 años. Este concepto, derivado del llamado silver market surgido en Japón (el país con mayor porcentaje de personas mayores de 65 años) durante la década de los setenta para referirse al mercado sénior que aglutina a sectores tan diversos como la salud, la banca, la automoción, la energía, la vivienda, las telecomunicaciones, el ocio y el turismo, entre otros (14).

Uno de los rasgos, entre otros, definitorios de los consumidores de la silver economy es que "Son personas activas a las que les gusta cuidarse, hacer deporte, comer bien, ir a la moda y divertirse (14).

Enfermería tiene mucho que decir en cuanto a personas mayores se refiere, ya que el campo de los cuidados es propio de la profesión y fortalecer emocionalmente a las personas, estimularlas para que tenga de una vida activa y saludable es uno de los objetivos. Fomentar una autoimagen positiva, elevando la autoestima es muy importante en la edad adulta, pues "sentirse guapo por fuera hace que se sienta uno guapo por dentro".

Zamora es la provincia más envejecida de España con una media de edad de 50,7 años supera en más de seis puntos a la media de edad nacional que es de 44,3 años, a fecha de 1 de enero de 2017 (14). Según estos datos Zamora sería la provincia "ideal" para ser un espacio Silver, en este caso de la moda para personas mayores (15).

El objetivo de nuestro estudio es evaluar la influencia de la moda en las personas mayores como potenciador de la autoestima, por lo tanto, como mejora de su salud y hacer de Zamora un referente de silverización del sector de la moda, adaptándose a los gustos y necesidades de las personas mayores. (Espacio fashion week de la moda Silver).

\section{MÉTODO}

Revisión bibliográfica de la literatura, con el objetivo de encontrar la mejor evidencia científica de cómo influye a nivel psicológico la moda en el ánimo o autoestima de las personas y así sentirse mejor, más valoradas, activas y guapas, factores importantes para prevenir la depresión, melancolía y otros. 
Se hace la búsqueda en distintas bases de datos, Pubmed, Cochrane, Cuiden, Cinhal, Scielo, Biblioteca Virtual de la Salud, Google Académico, utilizando los descriptores en términos DeCS: vestuario; anciano; Anciano de 80 o más Años; autoimagen y MeSH: clothing; aged; Aged, 80 and over; self concept.

Se utilizan como lenguaje libre las palabras: moda; fashion; chic; retail; autoestima.

Los criterios de inclusión son artículos que tengan que ver con la moda en ancianos o personas mayores.

Criterios de exclusión todos aquellos artículos que nos informan sobre moda en otros grupos de población que no sean ancianos y personas mayores.

No hay restricción de idioma ni de período de tiempo, ya que son escasos los artículos existentes.

Se localizan 69 artículos en Pubmed, siendo solo 1 el que reúne los requisitos del tema de interés de nuestra búsqueda. En el resto de bases no se ha encontrado ningún artículo.

El número de artículos ha sido muy escaso, 1 artículo en Pubmed, y 2 de búsqueda libre acordes al tema de búsqueda. Se encuentran bastantes artículos en tendencias de moda con piercing, tatuajes, etc. que no son relevantes para nuestra búsqueda

Se hace un barrido por Google para localizar cualquier tipo de información que sea objeto de nuestra investigación para poder aportar información para nuestra búsqueda.

El tema es muy novedoso, por lo tanto la búsqueda obtiene muy pocos resultados, resultando un tema de investigación futura.

\section{RESULTADOS}

En la revisión realizada por Saucedo et als. (16), concluye que las personas mayores están al día en las tendencias y compran prendas acordes a su estilo personal.

Las personas mayores son un mercado en aumento y no se están teniendo en cuenta ni sus deseos ni necesidades. El hecho de salir a comprar ropa, hace que se sientan felices satisfaciendo sus deseos, resultándoles gratificante y divertido y es percibido como placentero y lúdico.

Cada generación tiene una forma característica de ser, de vestir, de expresarse, trabajar, pensar, cuentan con un estilo propio que se tiene que tener en cuenta.

Comprar brinda placer, si es grata la percepción que se tiene de la tienda o centro comercial, de las características del producto, de la marca, personal que atiende, establecimiento, del horario de servicio, la ubicación del negocio, mobiliario, espacios, estacionamiento, etc., esto propicia a consumir proporcionando un alto grado de felicidad en las personas mayores.

Salir a comprar es una forma de relacionarse con las demás personas y permite formar su identidad y más cuando se trata de ropa, ya que imprime un estilo personal propio.

En marketing se deben conocer las necesidades del cliente objetivo para llegar a ellos certeramente. La moda expresa la cohesión del grupo hacia dentro y su diferenciación hacia fuera.

Comprar no solamente es destinar nuestros recursos, tiempo, entusiasmo y emoción a la obtención de lo que deseamos, sino que se estrechan las relaciones interpersonales y cada ser humano se imprime un sello especial que lo diferencia de los demás.

El estudio realizado por Karin Lovgren (17), entrevista a mujeres de 62 a 94 años para explorar los gustos de este grupo de edad, el resultado al que llega es que las preferencias de estilo, para vestir en este grupo de personas, es, ropa cómoda, práctica y que no exponga el cuerpo y sus cambios con el envejecimiento. Le dan importancia a la hora de sentirse a gusto y ropa adecuada para las ocasiones y situaciones. 


\section{MODA Y SALUD EN PERSONAS MAYORES. REVISIÓN BIBLIOGRÁFICA ENFERMERA}

El estudio realizado por Durá Gil et als, (18) y galardonado con el premio "Edad y vida" en 2006, presenta una visión sobre el estudio de la indumentaria en personas mayores y preferencias a la hora de la vestimenta; según datos que expone del INE (2004) los gastos destinados a indumentaria (ropa, calzado y cosmética) fueron de un $6,2 \%$, gastos que van en creciente aumento.

Las mujeres gastan más en indumentaria, pero se ve que en breve se igualará el gasto entre hombres y mujeres, ya que cada vez los hombres la dan mayor importancia a la imagen corporal.

Los principales problemas que encuentran las personas mayores con la indumentaria es que no se adecúan a sus necesidades, características y preferencias. La palabra confort en indumentaria para personas mayores lleva asociada negativamente el concepto de "ortopédico o para viejos"; colores austeros y diseños obsoletos.

Durá et als, exponen claramente que si se quiere llegar al mercado de las personas mayores, será necesario adaptarse a los gustos y comodidad de estilo de este grupo de personas. La indumentaria tendría que contar con las características de funcionalidad (confort, fácil de usar, adaptado a las dificultades físicas...) y estéticamente bonitas.

Los problemas que hay que tener en cuenta a la hora de diseñar ropa para personas mayores son, las deficiencias que puedan tener como, dificultad en los movimientos de los brazos, dificultad para abotonar camisas, dificultad para subir cremalleras, dificultad en la movilidad de piernas, dificultad para agacharse. En cuanto al calzado los problemas más comunes son la dificultad para abrochar 0 atarse los cordones 0 una horma inadecuada y dificultad para calzar.

Los tres autores coinciden en que, el grupo de personas mayores son un mercado en aumento, con rentas considerables, buena salud, interés por disfrutar, ilusiones y una estructura de consumo característica, el mercado tiene que dirigirse a ellos y considerar sus exigencias a la hora de diseñar y comercializar teniendo en cuenta sus gustos y preferencias, haciendo una moda cómoda y funcional para que se sientan bien $(16,17,18)$.

\section{DISCUSIÓN}

Son pocos los resultados obtenidos con el tema elegido de evaluar la influencia de la moda en las personas mayores como potenciador de la autoestima, por lo tanto, como mejora de su salud; ante este hecho vemos que es un tema novedoso y que indicaría investigaciones futuras sobre el tema, desde el punto de vista de la enfermería.

En otros tipos de pacientes, como pacientes con cáncer, son muchos los estudios que demuestran que el maquillaje, es muy importante a la hora de elevar la autoestima 0 autoimagen, y hace que se sientan mejor.

Enfermería cuenta con un Diagnóstico específico para la autoestima o autoimagen que es el codificado con el número 00120 y corresponde al diagnóstico "BAJA AUTOESTIMA SITUACIONAL", y entre otras intervenciones para este diagnóstico se encuentran, POTENCIACIÓN DE LA SOCIALIZACIÓN (5100), APOYO EMOCIONAL (5270), POTENCIACIÓN DE LA AUTOESTIMA (5400). Intervenciones que han quedado demostradas como válidas en el estudio que hemos realizado.

Como hemos explicado ampliamente en este revisión, el sentirse guapos, tener en cuenta la indumentaria para crear una "moda" para personas mayores, haría que muchas personas se sintiesen atractivas, guapas y por ende su autoestima estuviera más elevada.

Zamora es la ciudad más envejecida de España y probablemente de Europa, hagamos de ella una ciudad bonita para vivir, seamos referentes en moda para el espacio Senior o Silver, una ciudad con color en las calles, con habitantes que se sientan felices y guapos con la ropa que llevan ya que ser mayor no implica vestir de manera anticuada con colores austeros. La moda cómoda, fácil de llevar, colores que hagan sentirse atractivo y guapo hace que nos sintamos felices y por ello con buena percepción de la salud. 
Si te sientes feliz, te sentirás con más salud y esto está ampliamente demostrado.

\section{CONCLUSIÓN}

La profesión enfermera tiene en cuenta los gustos y preferencias de las personas, a la hora de establecer pautas para la afrontación de determinados estados de salud.

La autoimagen que uno tenga sobre sí mismo influye también en el estado de salud o enfermedad, si la percepción que tenemos de nosotros mismos es buena el estado de salud se percibirá como bueno o menos malo.

La imagen que vemos frente al espejo, es como vamos vestidos, arreglados la forma de combinar los colores, de utilizar los complementos, se sentirse cómoda y elegante, influye sobre la percepción de la salud y el bienestar dela persona.

"SIENTE GUAPA Y TE SENTIRÁS BIEN".

\section{REFERENCIAS BIBLIOGRÁFICAS}

Organización Mundial de la Salud. Envejecimiento y salud. [Sitio en Internet]. [Consultado el 11 de noviembre de 2020]. Disponible en: https://www.who.int/es/news-room/fact-sheets/detail/envejecimiento-y-salud

Durá Gil JV et als. Indumentaria de las personas mayores. Hábitos de compra y problemas de uso. Rev. Biomecánica. 2009; 52:35-38.

Enríquez G, Nájera S. Enfermería: fomento de la autoestima en padecimientos neuróticos en el adulto mayor. Rev. Actual de Costa Rica. [Internet]. 2014 [Citado el 19 de noviembre de 2020]; 26: 1-11. Recuperado a partir de: http://www.revenf.ucr.ac.cr/neurosis.pdf.ISSN 1409-4568

García, L. Autoestima en la personas mayores. [Internet]. 2019. [Citado el 21 de noviembre de 2020]. Recuperado a partir de https://www.cuidum.com/blog/autoestima/

Reynolds JR, Turner RJ. Major life events: their personal meaning, resolution, and mental health significance. J Health Soc Behav. 2008;49 (2):223-37.

Raich RM. Imagen corporal: conocer y valorar el propio cuerpo. Madrid: Editorial Pirámide; 2001.

Luna-Montaño I. Belleza, imagen corporal y psicopatología. Fuente: Interpsiquis 2004. Disponible en: http://www.psiquiatria.com/articulos/otras_enf_mentales/15091/

Lenze EJ. Comorbid anxiety disorders in depressed elderly patients. Am J Psychiatry. 2000;157 (5): 722-28.

Flint AJ. Epidemiology and comorbidity of anxiety disorders in the elderly. Am J Psychiatry. 1994; 151 (5):640-49

La importancia de cuidar la imagen en personas mayores. [Internet]. 2018. Inforesidencias. Recuperado a partir de:

https://www.inforesidencias.com/contenidos/noticias/nacional/la-importancia-de-cuidar-la-imagende-las-personas-mayores

La ropa que llevamos cambia nuestra manera de pensar. [Internet]. Tendencias21. 2014. Recuperado a partir de https://tendencias21.levante-emv.com/la-ropa-que-llevamos-cambianuestra-manera-de-pensar_a34284.html

La moda en la tercera edad. [Internet]. 2018. Bastón de Oro. Recuperado a partir de http://bastondeoro.com/la-moda-en-la-tercera-edad/

Parga M. Daphne Selfe, la modelo más anciana del mundo que será homenajeada por Isabel II. Vanity Fair. 6 de enero de 2019;35569. Disponible en: https://www.revistavanityfair.es /lujo/moda/articulos/modelo-mas-anciana-mundo-daphne-selfe-homenaje-reina-isabel/35569

'Silver economy', los mayores serán el motor de la economía del futuro. [Intenet] Iberdrola. Recuperado a partir de: https://www.iberdrola.com/innovacion/silver-economy 
Zamora, la provincia más envejecida de España. [Internet]. Noticialcyl. 2018. Recuperado a partir de https://www.noticiascyl.com/t/1753492/zamora-provincia-envejecida-espana.

Saucedo JM. Comportamiento que presenta la generación silenciosa respecto a la moda. Rev. Int. Admon \& financ. 2017;10(5):95-103.

Lovgren K.. Comfortable and leisurely: Old women on style and dress. J Women Aging. 2016; 28(5):372-85. 